\title{
Multiple stellar populations in Magellanic Cloud clusters
}

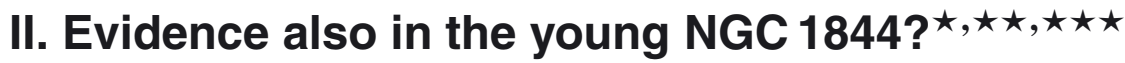

\author{
A. P. Milone ${ }^{1,2,3}$, L. R. Bedin ${ }^{4}$, S. Cassisi ${ }^{5}$, G. Piotto ${ }^{4,6}$, J. Anderson ${ }^{7}$, A. Pietrinferni ${ }^{5}$, and R. Buonanno ${ }^{5}$ \\ ${ }^{1}$ Instituto de Astrofîsica de Canarias, 38200 La Laguna, Tenerife, Canary Islands, Spain \\ e-mail: milone@iac.es \\ 2 Department of Astrophysics, University of La Laguna, 38200 La Laguna, Tenerife, Canary Islands, Spain \\ ${ }^{3}$ Research School of Astronomy and Astrophysics, The Australian National University, Cotter Road, Weston, ACT 2611, Australia \\ 4 INAF - Osservatorio Astronomico di Padova, Vicolo dell'Osservatorio 5, 35122 Padova, Italy \\ e-mail: luigi.bedin@oapd.inaf.it \\ 5 INAF - Osservatorio Astronomico di Collurania, via Mentore Maggini, 64100 Teramo, Italy \\ e-mail: [cassisi;pietrinferni; buonanno] @oa-teramo.inaf.it \\ 6 Dipartimento di Fisica e Astronomia "Galileo Galilei”, Università di Padova, Vicolo dell'Osservatorio 3, Padova, \\ 35122 Padova, Italy \\ e-mail: giampaolo.piotto@unipd.it \\ 7 Space Telescope Science Institute, 3800 San Martin Drive, Baltimore, MD 21218, USA \\ e-mail: jayander@stsci.edu
}

Received 15 October 2012 / Accepted 5 February 2013

\section{ABSTRACT}

\begin{abstract}
We used Hubble Space Telescope observations to study the young cluster NGC 1844 in the Large Magellanic Cloud (LMC). We estimated the fraction and the mass-ratio distribution of photometric binaries and report that the main sequence presents an intrinsic breadth which cannot be explained only in terms of photometric errors, and is unlikely because of differential reddening. We attempted different interpretations of this feature, including stellar rotation, binary stars, and the presence of multiple stellar populations of different ages, metallicity, helium, or $\mathrm{C}+\mathrm{N}+\mathrm{O}$ abundance. Although we have excluded age, helium, and $\mathrm{C}+\mathrm{N}+\mathrm{O}$ variations of being responsible for the main-sequence spread, none of the other interpretations is conclusive.
\end{abstract}

Key words. stars: Population II - globular clusters: individual: NGC 1844 - binaries: general - Magellanic Clouds

\section{Introduction}

High-accuracy photometry, mainly with the Hubble Space Telescope (HST), is revealing multiple sequences in the color-magnitude diagrams (CMDs) of a growing number of Galactic globular clusters (GGCs, e.g., Bedin et al. 2004; Anderson 1997; Piotto et al. 2007; Milone et al. 2008; Lee et al. 2009). Spectroscopy also shows that multiple stellar populations are a common feature among old GCs (e.g., Kraft et al. 1992; Yong et al. 2008; Marino et al. 2008; Carretta et al. 2009).

The presence of multiple stellar populations seems not to be an exclusive property of old stellar systems. Thanks to HST observations, the intermediate-age clusters (IACs) in the Magellanic Clouds (MCs) have also been found to host multiple stellar populations (Bertelli et al. 2003; Baume et al. 2007; Mackey \& Broby Nielsen 2007; Mackey et al. 2008; Glatt et al. 2008a,b; Goudfrooij et al. 2009, 2011).

It is now confirmed that over $70 \%$ (probably a lower limit set by the quality of the available data) of the $\sim 1-3$ Gyr old

* Based on observations with the NASA/ESA Hubble Space Telescope, obtained at the Space Telescope Science Institute, which is operated by AURA, Inc., under NASA contract NAS 5-26555, under GO-12219.

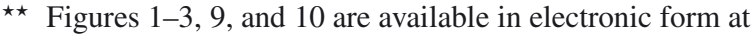
http://www . aanda.org

$\star \star \star$ Photometric data is only available at the CDS via anonymous ftp to cdsarc.u-strasbg. fr (130.79.128.5) or via

http://cdsarc.u-strasbg.fr/viz-bin/qcat?]/A+A/555/A143
MC clusters studied so far reveal some broadening of their sequences (Milone et al. 2009, hereafter Paper I), pointing to stellar populations with inhomogeneity in the chemistry, age, rotation, or other physical properties of their stars (see Keller et al. 2011, for a discussion).

It is worthwhile extending the study to stellar clusters younger than $\sim 300 \mathrm{Myr}$ to investigate whether the processes that generate broadened or multiple stellar sequences in the CMDs of GGCs and MC clusters are similar in nature or not, and to put on constraints when, after the cluster birth, these processes set in.

In this work we begin an investigation of young clusters, and search for the presence of multiple stellar populations among their stars. The target of the present study is NGC 1844, for which a summary of its main parameters is given in Table $1^{1}$. To our knowledge, the present study is the first attempt to extend the study of multiple populations to a $\sim 150$ Myr old cluster.

\section{Observations, measurements, and selections}

This work is based on coordinated parallel observations obtained with the Wide Field Channel (WFC) of the Advanced Camera

\footnotetext{
1 To obtain the core $\left(r_{\mathrm{c}}\right)$ and tidal radius $\left(r_{\mathrm{t}}\right)$ of NGC 1844, we first determined the center of the cluster using $2.5^{\prime \prime}$-bin-sized histograms along the $X$ and $Y$ directions for stars with an instrumental magnitude in $F 475 \mathrm{~W}$ brighter than -8 ; then we performed a least-square fit of the radial distribution of the number of stars to Eq. (14) in King (1962). The concentration, as defined by Harris (1996), is $c=\log \left(r_{\mathrm{t}} / r_{\mathrm{c}}\right)=0.4$, making NGC 1844 a loose cluster.
} 
Table 1. Parameters for NGC 1844 derived in this work.

\begin{tabular}{cccccccc}
\hline \hline$(m-M)_{F 814 W}$ & $E\left(m_{F 475 W}-m_{F 814 W}\right)$ & Age $(\mathrm{Myr})$ & $Z$ & {$[\mathrm{Fe} / \mathrm{H}]$} & Core radius $(\operatorname{arcsec})$ & Tidal radius $(\operatorname{arcsec})$ & Concentration \\
\hline 18.52 & 0.15 & 150 & 0.01 & -0.6 & 22 & 57 & 0.4 \\
\hline
\end{tabular}

for Surveys (ACS) at the focus of the HST under program GO-12219 (PI: Milone). The primary target of the program, entitled "Multiple stellar generations in the Large Magellanic Cloud Star Cluster NGC 1846", was indeed, NGC 1846, for which Wide Field Camera 3 observations were collected. At the phase II of GO-12219 we realized that the two clusters NGC 1846 and NGC 1844 were separated by the angular distance between the two far-most corners of the field of views of the two cameras: ACS/WFC and the UV and visual (UVIS) channel of WFC3, on the HST focal plane. We, therefore, decided to point and orient HST so as to collect images for both clusters simultaneously, in one shot (see Fig. 1).

This work is focused on NGC 1844, for which data were collected between 16 and 17 of April 2011, and consist of $7 \times 900 \mathrm{~s}$ images in filter $F 475 \mathrm{~W}$, and $1 \times 326 \mathrm{~s}+4 \times 340 \mathrm{~s}$ in $F 814 W$. A companion work will deal with NGC 1846. All images were dithered by whole and fractional pixels, as described in Anderson \& King (2000). Before performing measurements of the sources' positions and fluxes, we applied our recently developed pixel-based correction for imperfect charge transfer efficiency (CTE, Anderson \& Bedin 2010). Figure 2 shows a trichromatic stacked image of the studied ACS/WFC field ${ }^{2}$ after removal of cosmic rays and most of the artefacts. As can be seen, this choice of pointing allows for a proper estimate of the LMC field contamination. We will use this field to statistically correct the NGC 1844 CMD from field contamination.

Photometry and relative positions were obtained with the software tools described by Anderson et al. (2008). The photometry was calibrated into the WFC/ACS Vega-mag system following the procedures given in Bedin et al. (2005), and using the encircled energy and zero points given by Sirianni et al. (2005). We will use the symbols $m_{F 475 W}$ and $m_{F 814 W}$ for these calibrated magnitudes.

Artificial-star (AS) tests were performed using the procedures described by Anderson et al. (2008). In the present program we chose them to cover the magnitude range $20<$ $m_{F 475 W} \leq 30$, with colors that placed them on the main sequence (MS). Completeness has been calculated as in Paper I (see Sect. 2.2) and accounts for both the crowding conditions and stellar luminosity. Figure 3 shows the completeness contours in the radius versus magnitude plane.

Stars that saturate are treated as described in Sect. 8.1 in Anderson et al. (2008). Collecting photo-electrons along the bleeding columns allows us to measure magnitudes of saturated stars up to $\sim 3.5 \mathrm{mag}$ above saturation (i.e. up to $m_{F 814 W} \sim 20$, and $m_{F 475 W} \sim 20$ ), with errors of only a few percentage points (Gilliland 2004). We used $\sim 80$ sources in the 2 mass catalog to register our absolute astrometry. The calibrated catalog, and an astrometrized image has been released to the community as part of this work.

The analysis we present here requires high-precision photometry, so we selected a high-quality sample of stars that 1) have a good fit to the point-spread function; 2) are relatively

\footnotetext{
2 The color image is a trichromatic (RGB), where for the blue and the red channels we used the $F 475 W$ and $F 814 W$ stacks, and for the green channel we used a wavelength-weighted average of the two (using a weight of $3: 1$ ).
}

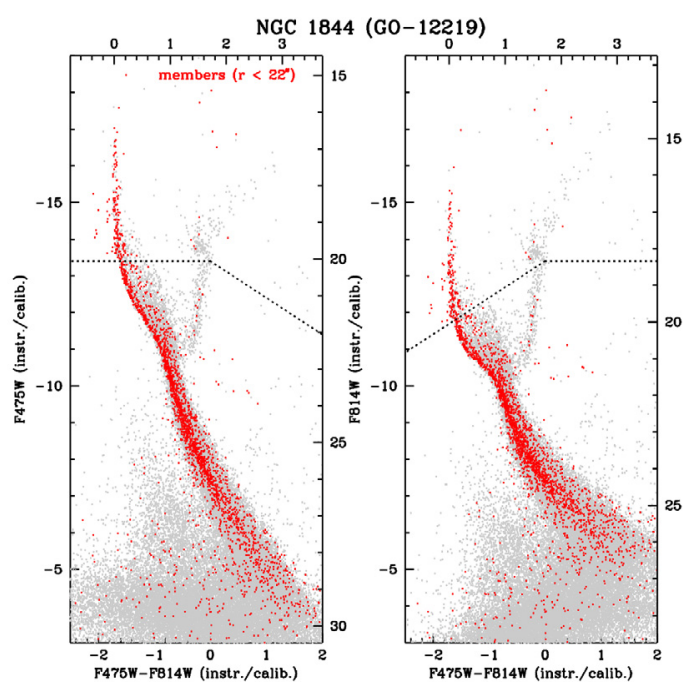

Fig. 4. CMDs for all the sources in the field (gray points). The objects within 22 arcsec from the assumed cluster center are shown as red dots. A dashed line indicates the set in of the saturations.

isolated; and 3) have small astrometric and photometric errors (see Paper I, Sect. 2.1 for a detailed description of this procedure).

\section{The color-magnitude diagrams}

Figure 4 shows the CMDs for all the detected sources in the studied field. The objects highlighted in red are those within 450 ACS/WFC pixels (i.e. 22", assuming a 49.7248 mas ACS/WFC-pixel scale, from van der Marel et al. 2007) from the assumed center of the cluster at $(\mathrm{RA} ; \mathrm{Dec})=(05: 07: 30.462$; $-67: 19: 27.79)$ (or in pixel-coordinates: $(4400 ; 1780)$ ).

A more careful inspection of the MS reveals some color structures. Figure 5 shows a quantitative analysis of this color structure. In the left panel we present the same $m_{F 814 W}$ versus $m_{F 475 W}-m_{F 814 W}$ CMD of Fig. 4 , with the fiducial line of the main MS component ${ }^{3}$ indicated and the loci (dashed line) occupied by the corresponding equal-mass binaries. A box indicates the region of the MS where this color-structure is most evident. For clearity, the top-right panel shows a blow-up of this box, without the fiducial line. The bottom-right panels show respectively, the "rectified MS" (obtained by subtracting the color of the fiducial from the color of each star at the corresponding magnitude) and the histogram of this distribution.

It is clear that the color distribution of the MS of NGC 1844 in this range of magnitudes has an anomalous skew towards red. We will see in the following that a very peculiar binary massratio distribution is required to reproduce the observations (see Sect. 3.2).

In the upper-left panel of Fig. 6 we plot the Hess diagram for this portion of the CMD for all objects within 22.3 arcsec from the assumed cluster center (hereafter cluster field). The upper-middle panel shows the Hess diagram for a field with radial distance from the cluster center $R>100$ arcsec, where no

\footnotetext{
3 The fiducial line was defined "by hand" (solid line).
} 

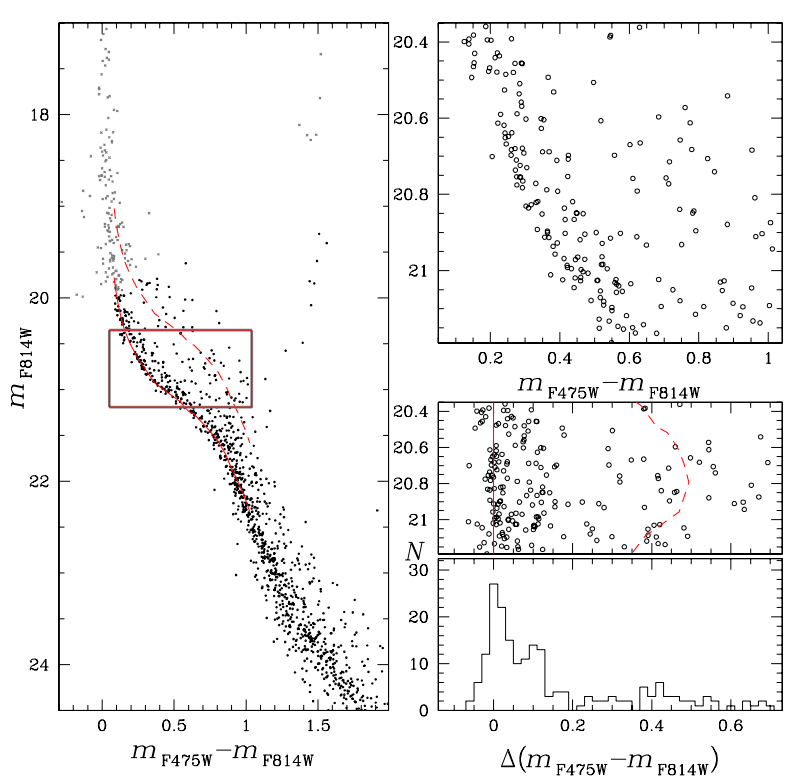

Fig. 5. On the left, the $m_{F 814 W}$ vs. $m_{F 475 W}-m_{F 814 W}$ CMD for stars in the cluster field. Saturated stars are colored gray. The solid and the dashed lines are the MS ridge line and the locus of equal-mass binaries, respectively. On the right, a zoom-in of the same CMD in the region highlighted by the box, i.e. where the MS broadening is most clearly visible (upper panel), the verticalized CMD (middle panel), and the color histogram distribution (lower panel) (see text).

cluster members are expected (see Fig. 2); we will refer to this as the reference field.

To statistically remove the contamination of field stars in the Hess diagram of stars in the cluster field, we compared the Hess diagrams of the cluster and the reference field. For each interval of color and magnitude used to make these two Hess diagrams, we calculated the number of cluster stars as $N_{\mathrm{CL}}=$ $N_{\mathrm{CF}}-f_{\mathrm{AREA}} N_{\mathrm{RF}}$, where $N_{\mathrm{CF}}$ and $N_{\mathrm{RF}}$ is the measured number of stars, corrected for completeness, in the cluster and reference field, respectively, $f_{\text {AREA }}$ is the ratio between the area of the cluster field and the area of the reference field. The Hess diagram of the cluster after that field's stars have been statistically removed is shown the upper-right panel. This plot demonstrates that the MS broadening cannot be explained by field contamination.

In the lower-left panel of Fig. 6 we show again a blow-up of a the same portion of the CMD for stars in the cluster field. To provide, a discrete-point example of the field contamination in the CMD of the cluster, the bottom-middle panel shows the CMD for stars within an area covering the same amount of sky as the cluster field. This was taken in an area away from NGC 1844, and within the reference field (see Fig. 2, outside the magenta circle); we will refer to this as the comparison field (in red in Fig. 2) and it will not be used for the quantitative analysis in this paper.

Finally, the lower-right panel shows the same CMD for the artificial stars added along the fiducial lines, after an additional broadening to account for the tendency of artificial star tests to underestimate photometric errors (see discussion in Paper I). The direct comparison of the two CMDs reveals an internal breadth of the MS of $\sim 0.1$ in color, which cannot be explained either by field contamination or by photometric errors.

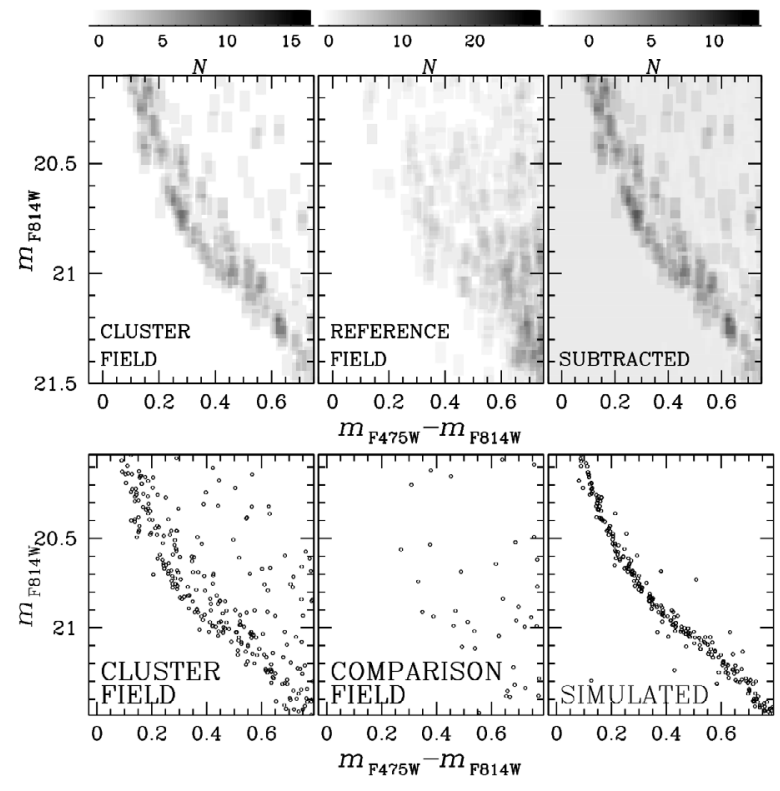

Fig. 6. Top panels: Hess diagrams around the anomalous MS breadth of NGC 1844 for the selected cluster field, the reference field, and for the area-normalized difference of the two. Bottom panels: CMD focused on the same region for the point sources measured in the cluster field, those measured in a comparison field, and for the photometry of ASs placed along the MS fiducial line.

\subsection{Simulation of binaries}

A visual inspection of the CMD of NGC 1844 reveals a large number of photometric binaries on the red side of the MS (Fig. 4). In this section we investigate whether these objects can account for the observed MS broadening.

To do this we started by deriving the fraction of MS-MS binaries with mass ratio $q>0.4$ by assuming that the MS broadening is due to binaries alone. Briefly, we divided the CMD into two parts: a region "A" populated by single stars and the binaries with a primary with $20.3<m_{F 814 W}<21.3$ (the shadowed area in the upper panels of Fig. 7), and a region " $\mathrm{B}$ " which is the portion of A containing the binaries with $q>0.4$ (the darker area in the upper panels of Fig. 7). The reddest line is the locus of the equal-mass binaries red-shifted by four $\sigma$ (where $\sigma$ is the error estimated as in Paper I). The bluest line is the MS fiducial moved by four $\sigma$ to the blue. The locus of the CMD of binaries with a given mass ratio has been determined by using the mass-luminosity relation of Pietrinferni et al. (2004).

The fraction of binaries with $q>0.4^{4}$ is calculated as in Eq. (1) in Milone et al. (2012) (repeated here for convenience):

$f_{\text {bin }}^{\mathrm{q}>0.4}=\frac{N_{\mathrm{REAL}}^{\mathrm{B}}-N_{\mathrm{FIELD}}^{\mathrm{B}}}{N_{\mathrm{REAL}}^{\mathrm{A}}-N_{\text {FIELD }}^{\mathrm{A}}}-\frac{N_{\mathrm{ART}}^{\mathrm{B}}}{N_{\mathrm{ART}}^{\mathrm{A}}}$,

where $N_{\mathrm{REAL}}^{\mathrm{A},(\mathrm{B})}$ is the number of stars in the cluster field (corrected for completeness) observed in region $\mathrm{A}(\mathrm{B})$ of the $\mathrm{CMD} ; N_{\mathrm{ART}}^{\mathrm{A},(\mathrm{B})}$ and $N_{\text {FIELD }}^{\mathrm{A},(\mathrm{B})}$ are the corresponding numbers of artificial stars and of stars observed in the reference field and normalized to the area

4 Note that the anomalous sequence is located in a region of the CMD populated by binaries with $0.4<q<0.6$. The cut at $q=0.4$ is chosen to investigate the possibility that this sequence is due to binary systems. In the magnitude interval $\sim 20.3-22.3$, the fiducial line made of binaries with $q=0.4$ is redshifted from the MS fiducial by roughly three times the color error, $\sigma$. 

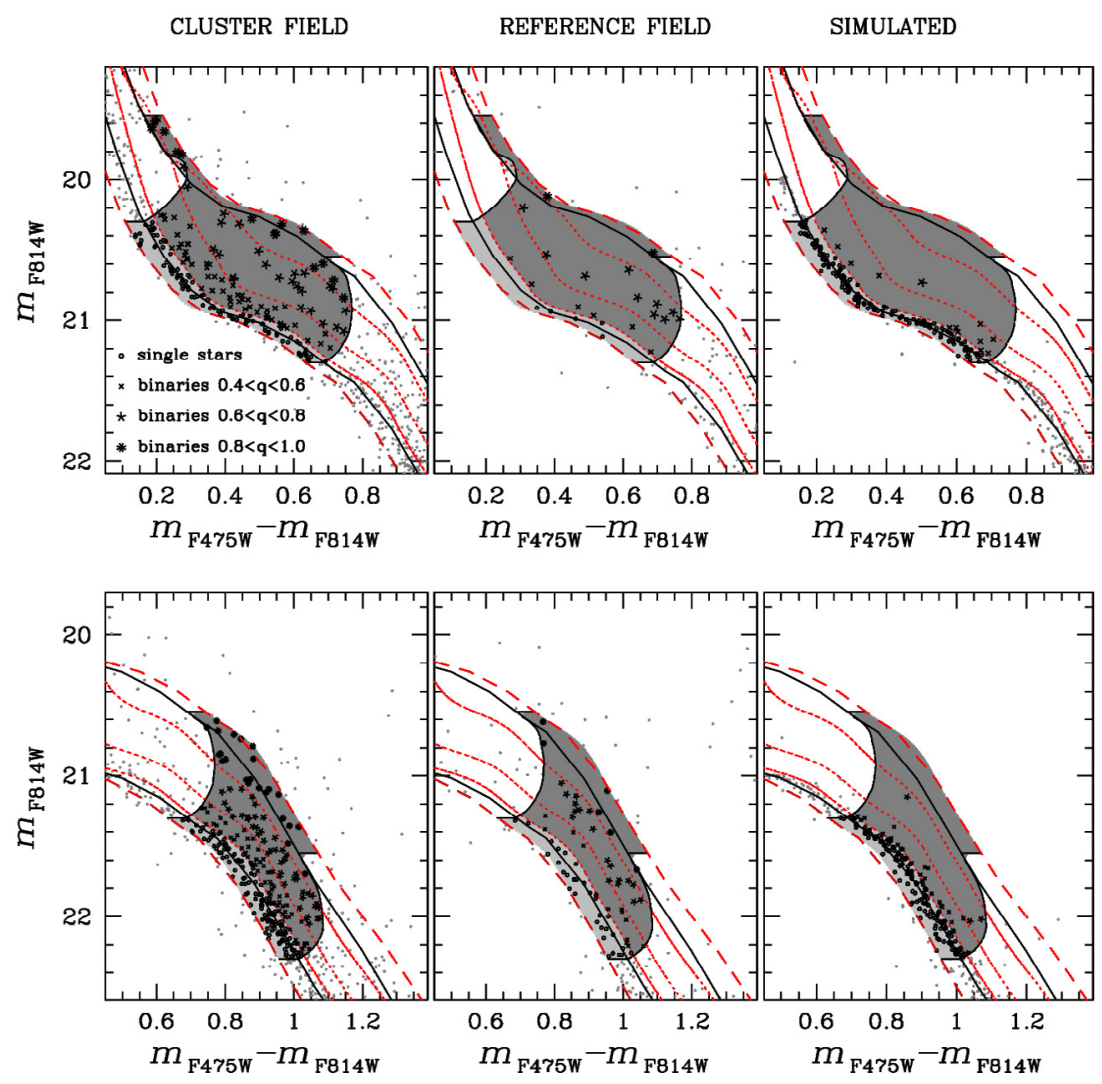

Fig. 7. CMD of stars in the cluster field (left), the comparison field (middle), and simulated stars (right). The continuous black lines are the MS fiducials and the locus of equal-mass binaries, while the loci of binaries with mass ratio $q=0.4,0.6$, and 0.8 are represented with dotted red lines (see text for more details). Upper and lower panels show the setup used to measure the fraction of binaries in the intervals $20.3<m_{F 814 W}<$ 21.3 and $21.3<m_{F 814 W}<22.3$ respectively.

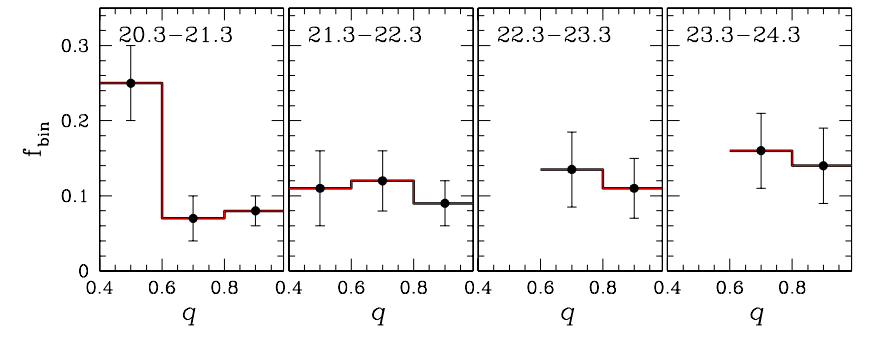

Fig. 8. Histogram distributions of the fraction of binaries as a function of the mass ratio, in four $m_{F 814 W}$ intervals indicated in each panel.

of the cluster field. We find $f_{\text {bin }}^{\mathrm{q}>0.4}=0.39 \pm 0.05$. We repeated the same procedure for the fraction of binaries with $q>0.6$ and $q>0.8$ and derived the fraction of binaries in three intervals of size $\Delta q=0.2$ in the interval $0.4<q<1$.

In the magnitude interval $20.3<m_{F 814 W}<21.3$, the fractions of binaries with $0.8<q<1$ and $0.6<q<0.8$ are similar $(0.10 \pm 0.03$ and $0.07 \pm 0.03$, respectively), but we need $23 \pm 5 \%$ of binaries with $0.4<q<0.6$ to account for the MS broadening. Results are plotted in Fig. 8.

To further investigate the influence of binaries on the MS morphology, we analyzed the CMD region with $21.3<$ $m_{F 814 W}<22.3$ where there is no evidence for an intrinsic color spread. Results are illustrated in the lower panels of Fig. 7. By using the procedure described above, we find $f_{\text {bin }}^{q>0.4}=0.30 \pm$ 0.05 and in this case each of the three analyzed mass ratios contains about $10 \%$ of the binaries.
For completeness we extended the study of binaries to fainter magnitudes. The binary fractions in the magnitude intervals $22.3<m_{F 814 W}<23.3$ and $23.3<m_{F 814 W}<24.3$ are listed in Table 2 and are plotted against $q$ in Fig. 8. Because of to the rise in the photometric error, the color distance of binaries with $q<0.6$ from the MS fiducial is smaller than three times $\sigma$, making it not possible to distinguish them from single MS stars. In these cases we limited our study to binaries with mass ratio $q>0.6$.

In addition, we repeated the same analysis described above using the $m_{F 475 W}$ versus $m_{F 475 W}-m_{F 814 W}$ CMD. We analyzed four $F 475 \mathrm{~W}$ intervals that correspond to the four $F 814 \mathrm{~W}$ bins previously defined and obtained similar results, which are listed in Table 2. We emphasize that, because of the relatively small number of binaries and the statistical approach used to subtract the background, any conclusion on the flatness of the $q$-distribution could be an over-interpretation of the data.

In summary, our investigation is not conclusive; the MS broadening could be due to binaries, but this hypothesis would imply an ad hoc mass-ratio distribution for the photometric binaries, with a large fraction of them concentrated in the interval of magnitude $20.3<m_{F 814 W}<21.3$ and mass ratio $0.4<q<0.6$.

\subsection{Differential reddening}

The extinction due to Galactic interstellar medium (ISM) in the direction of NGC 1844 is $E(B-V)=0.05$ (Schlegel et al. 1998) and such a small reddening is usually uniform over a scale of few arcsec. As an example, in Paper I we showed that the CMD 
of NGC 1846, a cluster in the LMC located about 5 arcmin S-W from NGC 1844, reveals a very narrow RGB and a well defined red clump, suggesting that any reddening variation in the area should be very small $(E(B-V)<0.007)$. The color difference between stars on the blue and red side of the MS of NGC 1844 is typically $\Delta \sim 0.07 \mathrm{mag}$ and is too large to be explained in terms of reddening variations only, as these variations would be larger than the average reddening itself $(E(B-V) \sim 0.05 \mathrm{mag})$.

To investigate whether differential reddening is responsible for the MS broadening of NGC 1844 we applied the procedure illustrated in Fig. 9. In the upper-left panel, we selected two groups of bona fide blue MS and red MS stars in the cluster field's CMD that we color coded blue and red, respectively. The same colors are used consistently in the other panels.

The upper-right panel shows that the spatial distribution of blue and red MS stars is the same (within the statistical uncertainties). We then divided the cluster field in four parts (quadrants) and plotted the corresponding CMDs in the lower panels. The numbers of blue MS and red MS stars are labeled in the lower-left corner of each CMD panel, and give the same ratio of red-to-blue stars within $1 \sigma$. However, we note that the small number of stars prevents us from a more significant analysis of the inference of reddening variations on shorter angular scales.

The present analysis suggests that differential reddening produced by Milky Way ISM is unlikely to be responsible for the MS broadening of NGC 1844. However we can not exclude that differential absorption due to the possible presence of intra-cluster nebulosity could generate this effect.

The cumulative radial distributions of blue MS and red MS stars are shown in Fig. 10. The Kolmogorov-Smirnov statistic shows that in random samplings from the same distribution a difference this large would occur $89 \%$ of the time, which is very reasonable for the hypothesis that the two MSs have the same distribution.

\section{Comparison with theory}

To understand the physical reasons of the MS broadening discussed in the previous sections, we have performed qualitative comparisons of observations with theoretical predictions taken from the BaSTI archive ${ }^{5}$. In doing this we employed both the $\alpha$-enhanced and the CNO-enhanced stellar models (Pietrinferni et al. 2004, 2006, 2009), specifically transformed into the ACS/WFC photometric Vega-mag system (Bedin et al. 2005). The adopted evolutionary stellar models account for the occurrence of core convective overshoot during the central H-burning stage by adopting the numerical assumptions and formalism discussed in Pietrinferni et al. (2004).

These comparisons are in the form of best fits to the observed CMD of NGC 1844 with isochrones calculated under different scenarios, which are discussed in the following subsections where we investigate the effect of CNO, metallicity, and helium variation, as well as the effect of stellar rotation.

\section{1. $\mathrm{CNO}$}

The top-left panel of Fig. 11 shows that an $\alpha$-enhanced isochrone for $Z=0.01$ and age of $150 \mathrm{Myr}$ (solid line) reproduces finely the morphology of the bulk of the MS as well as the brightness of the cluster turnoff. (Hereafter, unless otherwise specified, we adopt $(m-M)_{F 814 W}=18.52 \mathrm{mag}$ and $E\left(m_{F 475 W}-m_{F 814 W}\right)=$ $A_{F 475 W}-A_{F 814 W}=0.15$.)

\footnotetext{
http://wWw. oa-teramo.inaf.it/BASTI
}

Table 2. Fraction of binaries for different luminosity and mass-ratio intervals.

\begin{tabular}{cccc}
\hline \hline Luminosity bin & \multicolumn{3}{c}{$f_{\text {bin }}$} \\
\hline$F 814 W$ & $0.4<q<0.6$ & $0.6<q<0.8$ & $0.8<q<1.0$ \\
\hline $20.3-21.3$ & $0.25 \pm 0.05$ & $0.07 \pm 0.03$ & $0.08 \pm 0.02$ \\
$21.3-22.3$ & $0.11 \pm 0.05$ & $0.12 \pm 0.05$ & $0.08 \pm 0.04$ \\
$22.3-23.3$ & - & $0.13 \pm 0.05$ & $0.11 \pm 0.04$ \\
$23.3-24.3$ & - & $0.16 \pm 0.05$ & $0.14 \pm 0.05$ \\
\hline$F 475 W$ & $0.4<q<0.6$ & $0.6<q<0.8$ & $0.8<q<1.0$ \\
$20.46-21.99$ & $0.23 \pm 0.05$ & $0.05 \pm 0.03$ & $0.10 \pm 0.03$ \\
$21.99-23.31$ & $0.08 \pm 0.05$ & $0.11 \pm 0.04$ & $0.09 \pm 0.03$ \\
$23.31-24.57$ & - & $0.13 \pm 0.05$ & $0.15 \pm 0.04$ \\
$24.57-25.93$ & - & $0.17 \pm 0.05$ & $0.17 \pm 0.05$ \\
\hline
\end{tabular}

The same figure also shows that an isochrone based on stellar models with a factor of $\sim 2$ enhancement in the CNO-element sum is able to match the red boundary of the MS locus. So, the observed MS broadening could be due to a spread in the $(\mathrm{C}+\mathrm{N}+\mathrm{O})$ among stars in NGC 1844.

However, the brighter portion of the CNO-enhanced isochrones crosses a region of the CMD where no stars are observed. So, only an ad hoc mass function for the $(\mathrm{C}+\mathrm{N}+\mathrm{O})$-enhanced component could reconcile this scenario with the observations.

\subsection{Metallicity}

As an alternative scenario, we explored the possibility that the MS-broadening could be due to an intrinsic metallicity spread: the top-right panel of Fig. 11 shows that the color spread of the MS locus in NGC 1844 is confined within the theoretical predictions provided by two isochrones with metallicity $Z=0.01$ and 0.015 . This means that a metallicity spread of the order of 0.15-0.20 dex could account for the observed MS's broadening. Most importantly, this scenario is able to provide a better match than the previous one to the observed star distribution in the brightest portion of the CMD, i.e. $18.5<m_{F 814 W}<20.5$.

\subsection{Helium}

It is commonly accepted that the MS broadening (or split) observed in many GGCs hosting multi-populations (such as $\omega$ Cen, NGC 2808, NGC 6752, and 47 Tuc) is mainly due to a significant helium-abundance enhancement. However, as we will see, this scenario seems not to work to explain the MS broadening of NGC 1844. The bottom-left panel of Fig. 11 shows the comparison between the empirical data and selected isochrones for a fixed metallicity $(Z=0.008)$ and two different assumptions about the initial He content $(Y=0.256$ and $Y=0.300)$. In this case we had to adopt a shorter distance modulus of $(m-M)_{F 814 W}=18.40 \mathrm{mag}$. The He-enhanced isochrone matches the hotter boundary of the MS locus corresponding to the bulk of the cluster stellar population, while the isochrone corresponding to the "canonical" He abundance is not able to properly match the cooler edge of the MS locus. The agreement could improve by using a larger He enhancement $(Y \approx 0.33)$, but at the expense of an even smaller distance modulus a choice not supported by current best estimates of the LMC distance (e.g., Tammann et al. 2008, and references therein). 


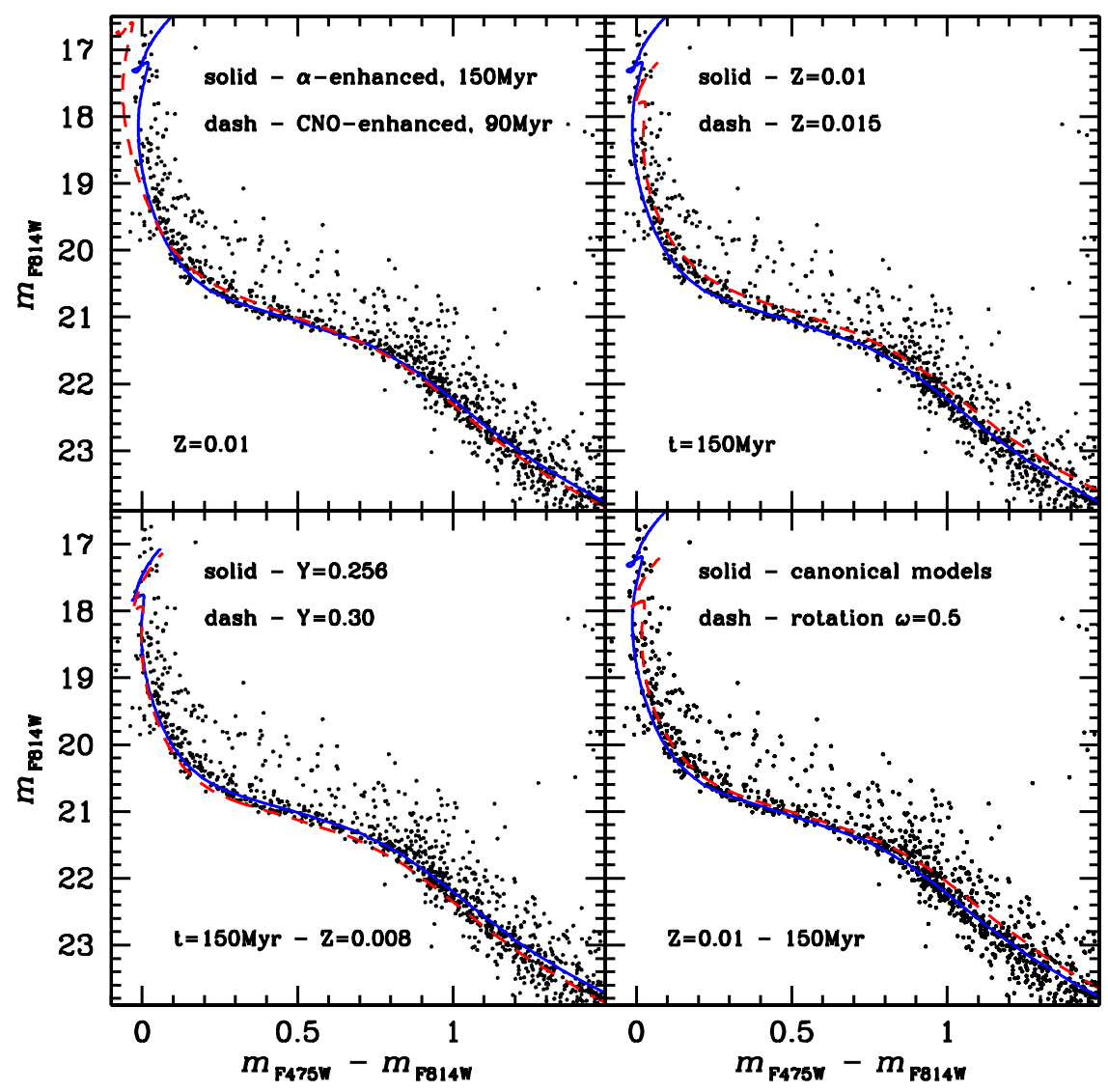

Fig. 11. Top left: comparison between the observed CMD of NGC 1844 and suitable theoretical isochrones for an age of $150 \mathrm{Myr}$ and $90 \mathrm{Myr}$ for two different assumptions about the heavy-element mixtures, an $\alpha$-enhanced mixture ( $[\alpha / \mathrm{Fe}]=+0.4$, solid blue line), and a CNO-enhanced mixture (dashed red line), both with the same iron content $[\mathrm{Fe} / \mathrm{H}]=-0.6$. In this comparison we assumed $(m-M)_{F 814 W}=18.52$ and $E\left(m_{F 475 W}-m_{F 814 W}\right)=$ 0.15 for both isochrones. Top-right: in this case the two isochrones have the same $\alpha$-enhanced mixture but different metallicities, namely $Z=0.01$ and $Z=0.015$. Bottom left: in this case the two isochrones have the same metallicity, $Z=0.008$, but two different initial He abundances. In this last case we adopted $(m-M)_{F 814 W}=18.40$. Bottom-right: comparison between the NGC $1844 \mathrm{MS}$ and the same isochrone ( $\alpha$-enhanced, $150 \mathrm{Myr}$, $Z=0.01$ ) adopted in the top left panel (solid blue line). To investigate the impact of stellar rotation, we also show the same isochrone modified according to the Bastian \& De Mink (2009) recipes to take into account the effect of rotation (dashed red line). (See text for more detail).

\subsection{Rotation}

As a last hypothesis, we explore another physical process that could help explain the observed MS broadening of NGC 1844: rotation of stars. This process is certainly able to affect both the evolutionary lifetimes and the morphology of the evolutionary tracks (Maeder \& Meynet 2000). Briefly, the centrifugal acceleration reduces the effective gravity resulting in cooler and slightly less luminous stars. However, rotation also induces internal mixing processes, which can have the opposite effect, i.e., leading to more luminous and hotter stars. Which of these contrasting effects dominates depends on the initial mass, rotational velocity, and chemical composition.

It has been suggested that the presence of fast rotators among MS stars could be the cause of the occurrence of multiple MS turn-offs in several IAC of the MCs (Bastian \& De Mink 2009). Although Girardi et al.(2011) compared isochrones from models with and without rotation with the observed CMDs and excluded this possibility, stellar rotation is a good candidate to explain the broadening of the MS locus in clusters as young as NGC 1844.

In the intermediate-mass regime - the one relevant to the present investigation (i.e., 150-Myr-young clusters) - and for stars still in the core H-burning stage, the dominant effect induced by rotation is the reduction of both the effective temperature and luminosity with respect to non-rotating stellar structures. Therefore, the existence of a spread in the rotational rate among the stars of NGC 1844 could help to explain the MS breadth.

A detailed investigation of stellar rotation is beyond the aims of the present work, therefore, to quantify the effect of stellar rotation on our isochrones, we have adopted a simplified approximation.

In the bottom-right panel of Fig. 11 we compare a selected portion of the NGC 1844 CMD (where the MS broadening is more evident) with two isochrones. The first one (solid blue line) is the same isochrone as adopted in the top-left panel of Fig. 11 ( $\alpha$-enhanced, $Z=0.01$ ). The second one (dashed red line) is the same isochrone after modifying its effective temperature and luminosity to take into account the effect of rotation. To account for rotation, we followed the detailed recipes given in Bastian \& De Mink (2009) ${ }^{6}$. According to their formalism, we adopted the

6 We note that Girardi et al. (2011) suggested that the approach by Bastian \& De Mink (2009) represents a too crude approximation to mimic the effects of stellar rotation because this simplification does not take into account the effect of rotation on the evolutionary timescale. However, we are limiting our analysis here only to the non-evolved stars along the MS, so we are interested just in obtaining an estimate of the changes in color and brightness induced by rotation. 
A. P. Milone et al.: Multiple stellar populations in Magellanic Cloud Clusters. II.

following values for the relevant parameters: $a=0.18, b=0.5$, and $\omega=0.5$. Although we are aware that this approach is extremely simplified, the data shown in the quoted figure reveal that the presence of a spread in the rotational rates can help to explain the MS broadening.

Whatever the reason of the intrinsic breadth of the MS of NGC 1844, we have demonstrated that it is significantly broader than can be expected from photometric errors and, therefore, real. Further investigation, from both a theoretical and an observational point of view, should be pursued.

Acknowledgements. We thank the referee for the constructive report that significantly improved the quality of this manuscript. A.P.M. acknowledges the financial support from the Australian Research Council through Discovery Project grant DP120100475. G.P. acknowledges support from the Università di Padova, grant CPDA101477. SC acknowledges financial support from PRIN INAF "Formation and Early Evolution of Massive Star Clusters". S.C. and R.B. also acknowledge financial support from PRIN MIUR 2010-2011, project "The Chemical and Dynamical Evolution of the Milky Way and Local Group Galaxies", prot. 2010LY5N2T. Support for this work has been provided by the IAC (grant 310394), and the Education and Science Ministry of Spain (grants AYA2007-3E3506 and AYA2010-16717).

\section{References}

Anderson, A. J. 1997, Ph.D. Thesis, Univ. of California, Berkeley Anderson, J., \& King, I. R. 2000, PASP, 112, 1360

Anderson, J., \& Bedin, L. R. 2010, PASP, 122, 1035

Anderson, J., Sarajedini, A., Bedin, L. R., et al. 2008, AJ, 135, 2055

Bastian, N., \& De Mink, S. E. 2009, MNRAS, 398, L11

Baume, G., Carraro, G., Costa, E., Méndez, R. A., \& Girardi, L. 2007, MNRAS, 375, 1077

Bedin, L. R., Piotto, G., \& Anderson, J., et al. 2004, ApJ, 605, L125

Bedin, L. R., Cassisi, S., Castelli, F., et al. 2005, MNRAS, 357, 1038
Bedin, L. R., Salaris, M., Piotto, G., et al. 2008, ApJ, 679, L29

Bertelli, G., Nasi, E., Girardi, L., et al. 2003, AJ, 125, 770

Carretta, E., Bragaglia, A., Gratton, R. G., et al. 2009, A\&A, 505, 117

D’Ercole, A., D’Antona, F., Carini, R., Vesperino, E., \& Ventura, P. 2012, MNRAS, 423, 1521

Gilliland, R. 2004, ACS Instrument Science Report, 2004-01

Girardi, L., Eggenberger, P., \& Miglio, A. 2011 MNRAS, 412, L103

Glatt, K., Grebel, E. K., Sabbi, E., et al. 2008a, AJ, 136, 1703

Glatt, K., Grebel, E. K., Sabbi, E., et al. 2008b, AJ, 135, 1106

Goudfrooij, P., Puzia, T. H., Kozhurina-Platais, V., \& Chandar, R. 2009, AJ, 137, 4988

Goudfrooij, P., Puzia, T. H., Kozhurina-Platais, V., \& Chandar, R. 2011, ApJ, 737, 3

Keller, S. C., Mackey, A. D., \& Da Costa, G. S. 2011, ApJ, 731, 22

Kraft, R. P., Sneden, C., Langer, G. E., \& Prosser, C. F. 1992, AJ, 104, 645

King, I. 1962, AJ, 67, 471

Mackey, A. D., \& Broby Nielsen, P. 2007, MNRAS, 379, 151

Mackey, A. D., Broby Nielsen, P., Ferguson, M. N., \& Richardson, J. C. 2008, ApJ, 681, L17

Maeder, A., \& Meynet, G. 2000, ARA\&A, 38, 143

Lee, J.-W., Kang, Y.-W., Lee, J., \& Lee, Y.-W. 2009, Nature, 462, 480

Marino, A. F., Villanova, S., Piotto, G., et al. 2008, A\&A, 490, 625

Milone, A. P., Bedin, L. R., \& Piotto, G. et al. 2008, ApJ, 673, 241

Milone, A. P., Bedin, L. R., Piotto, G., \& Anderson, J. 2009, A\&A, 497, 755

Milone, A. P., Piotto, G., Bedin, L., R., et al. 2012, A\&A, 540, A16

Pietrinferni, A., Cassisi, S., Salaris, M., \& Castelli, F. 2004, ApJ, 612, 168

Pietrinferni, A., Cassisi, S., Salaris, M., \& Castelli, F. 2006, ApJ, 642, 797

Pietrinferni, A., Cassisi, S., Salaris, M., Percival, S., \& Ferguson, J. W. 2009, ApJ, 697, 275

Piotto, G. 2009, IAU Symp., 258, 233

Piotto, G., Bedin, L. R., \& Anderson, J., et al. 2007, ApJ, 661, L53

Tammann, G. A., Sandage, A., \& Reindl, B. 2008, ApJ, 679, 52

Schlegel, D. J., Finkbeiner, D. P., Davis, M. 1998, ApJ, 500, 525

Sirianni, M., Jee, M. J., Benítez, N., et al. 2005, PASP, 117, 1049

Yong, D., Grundahl, F., Johnson, J. A., \& Asplund, M. 2008, ApJ, 684, 1159

van der Marel, R. P. 2003, Instrument Science Report ACS 2003-10, 10 
A\&A 555, A143 (2013)
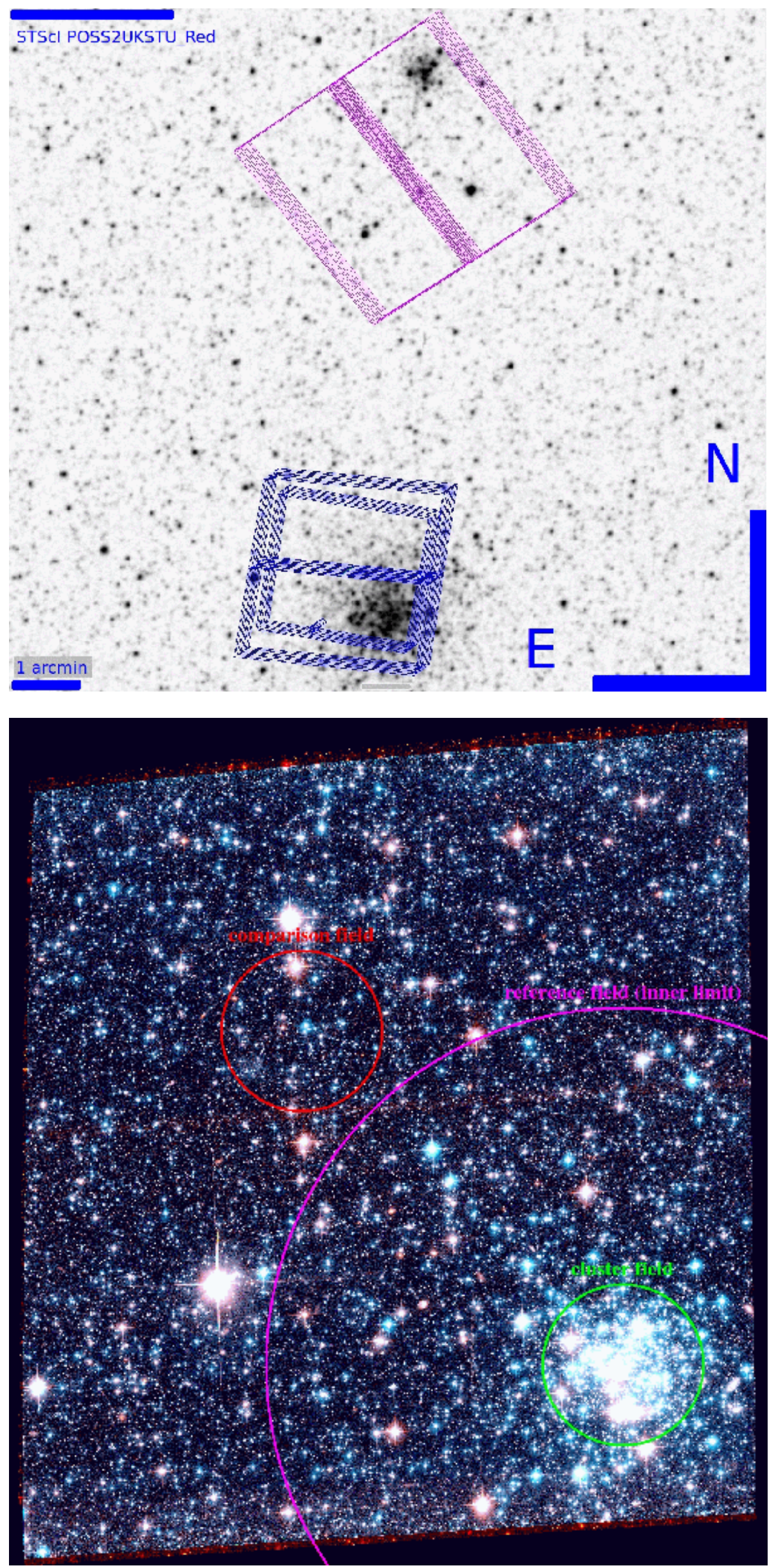

Fig. 1. Layout of the simultaneous observations of NGC 1846 (bottom) and NGC 1844 (top) (from the HST Astronomer's Proposal Tool). The individual ACS/WFC images are shown in magenta, and those of WFC3 (in both UVIS and NIR channels) in blue. Note the intrinsic different size of the two clusters, as they are at almost the same distance.
Fig. 2. Stacked trichromatic image of the studied NGC 1844 ACS/WFC-field. The green circle indicates the considered cluster region (cluster field), while the red circle is a random region of equal size shown for comparison (comparison field). The magenta circle indicates the inner limit of the portion of the ACS/WFC field used as reference to statistically remove the LMC field contamination from the CMD of NGC 1844. 
A. P. Milone et al.: Multiple stellar populations in Magellanic Cloud Clusters. II.

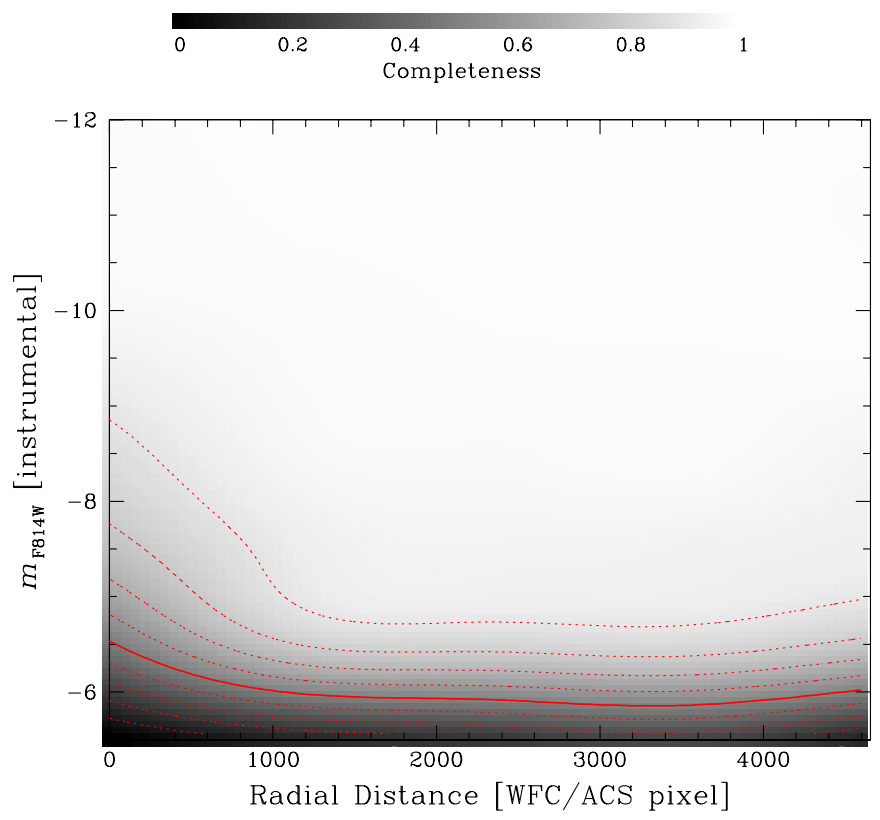

Fig. 3. Completeness contours in the radial-distance versus $m_{F 814 W}$ magnitude plane. The completeness is proportional to the gray level as indicated by the scale ot the top. Continuous lines correspond to a completeness level of 0.50 . Dotted lines indicate differences of completeness of 0.10 .
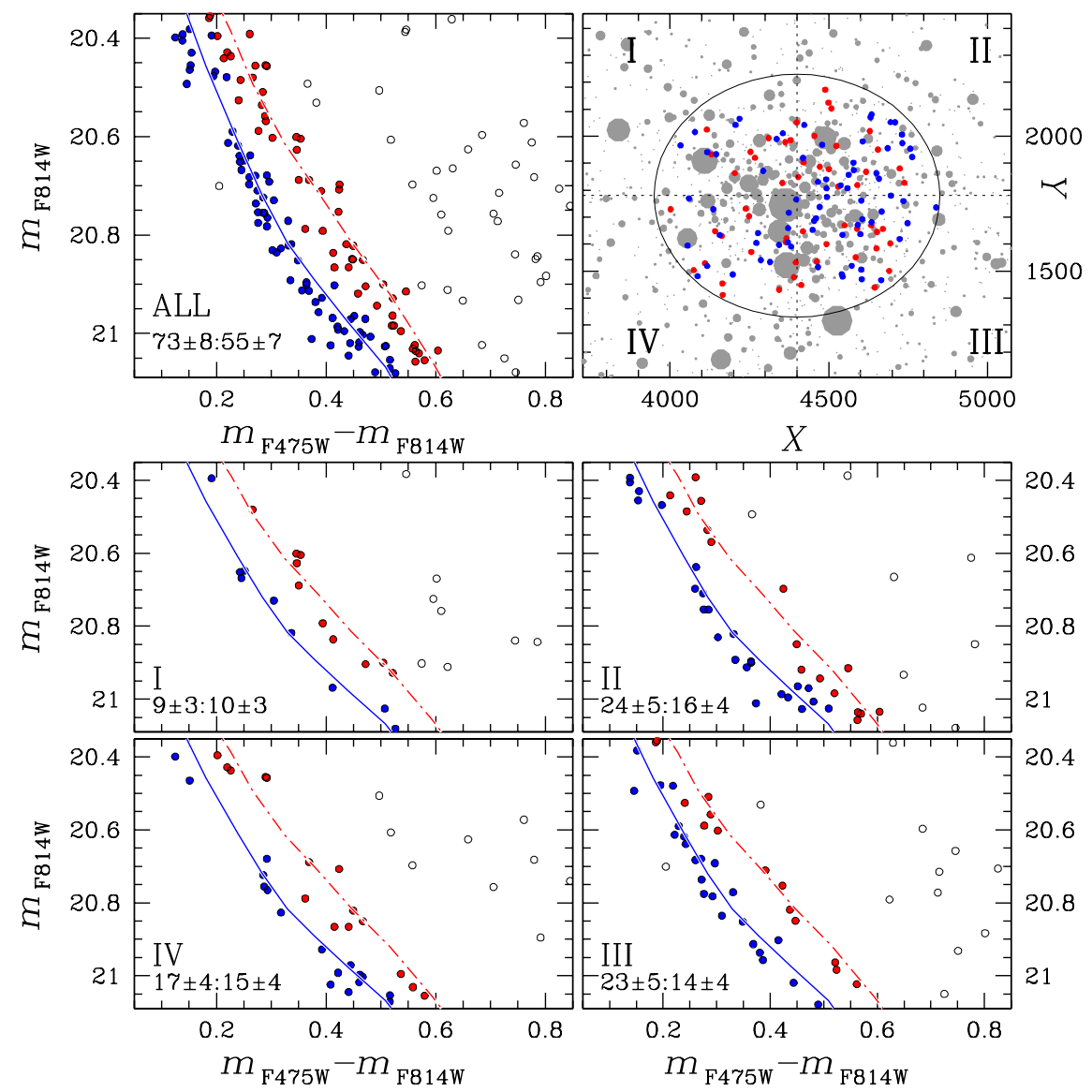

Fig. 9. Upper-left panel: CMD of stars in the cluster field zoomed-in around the region where the MS broadening is more evident. Stars on the blue and the red MS side are color coded in blue and red, respectively. The continuous and the dashed-dotted lines are the fiducial of the two MS regions drawn by hand. Upper-right panel: spatial distribution of blue and red MS stars. Lower panels: CMDs for stars in the four quadrants defined in the upper-right panel. 
A\&A 555, A143 (2013)

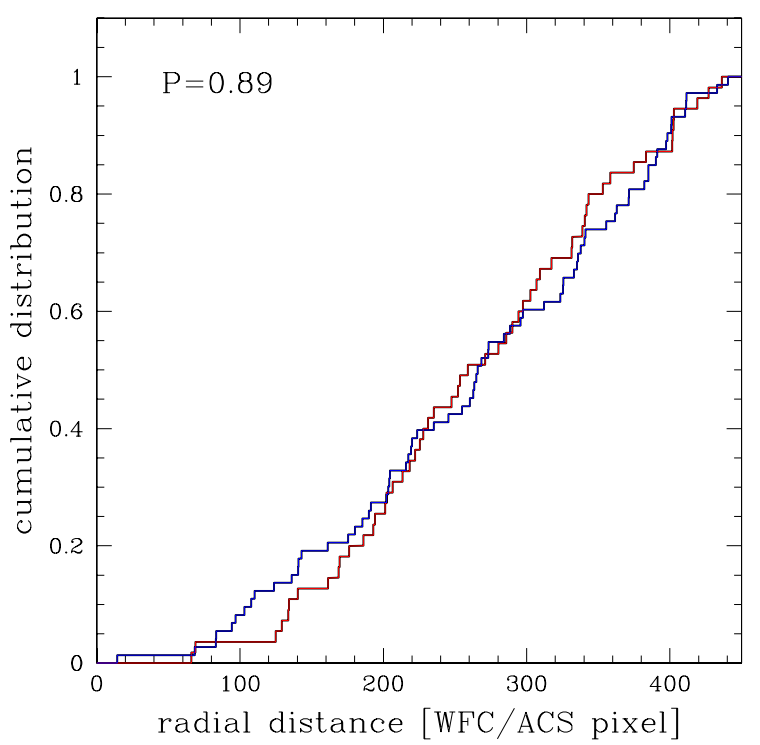

Fig. 10. Cumulative radial distribution of blue MS (blue line) and red MS (red line). 